\title{
Análise e classificação da rugosidade palatina em um grupo de jovens adultas
}

\section{brasileiras}

\author{
Analysis and classification of palatal rugae patterns in a group of young brazilian adults \\ Análisis y clasificación de la rugosidad palatina en un grupo de jóvenes adultas brasileñas
}

Recebido: 06/01/2021 | Revisado: 14/01/2021 | Aceito: 20/01/2021 | Publicado: 24/01/2021

\author{
Ana Beatriz Manetti Manganotti \\ ORCID: https://orcid.org/0000-0001-8948-3368 \\ Universidade Cesumar de Maringá, Brasil \\ E-mail: beatriz.manganotti@hotmail.com \\ Nicole Cordeiro Faria \\ ORCID: https://orcid.org/0000-0002-8364-3161 \\ Universidade Cesumar de Maringá, Brasil \\ E-mail: nicolecordeirof@yahoo.com.br \\ Fernanda Darlene Franzak \\ ORCID: https://orcid.org/0000-0003-0553-6464 \\ Universidade Cesumar de Maringá, Brasil \\ E-mail: fernanda_franzak@ @otmail.com \\ Marcelo Augusto Amaral \\ ORCID: https://orcid.org/0000-0001-6325-9210 \\ Universidade Cesumar de Maringá, Brasil \\ E-mail: marcelo.amaral@unicesumar.edu.br
}

\begin{abstract}
Resumo
Objetivo: Analisar a rugosidade palatina de um grupo de jovens adultas brasileiras, classificar por meio da técnica rugoscópica esta amostra, bem como avaliar possíveis diferenças entre faixas de idade, cor da pele, uso de aparelho ortodôntico e realização de cirurgias bucais nas pesquisadas. Material e Métodos: Estudo observacional, classificatório e transversal, com abordagem quantitativa das rugas do palato. A amostra de conveniência foi obtida a partir de 30 universitárias do sexo feminino do curso de Odontologia de uma instituição privada. A coleta dos dados foi realizada por meio de fotografias intrabucais do palato de cada uma das participantes, sendo realizado um destaque das rugas palatinas com auxílio do Microsoft Paint ${ }^{\circledR}$, com posterior classificação das imagens por uma única pesquisadora. Para a classificação das rugas palatinas das universitárias, levou-se em consideração o método proposto por Carrea (1937). Os dados foram analisados estatisticamente com a utilização dos testes Qui-Quadrado, Exato de Fischer e Razão de Verossimilhança $(\mathrm{p}=0,05)$. Resultados: Houve predominância de mulheres com faixa de idade de 19 a 22 anos $(70,0 \%)$, leucoderma $(83,3 \%)$, que fizeram uso de aparelho ortodôntico $(83,3 \%)$ e negaram ter realizado qualquer tipo de cirurgia bucal $(56,7 \%)$. O estudo identificou de sete a 18 rugas palatinas, com variados formatos. Conclusão: Houve maior incidência de rugas com direção em sentidos variados (tipo IV). A rugoscopia palatina não resultou em caracterizações com diferenças etárias, de ancestralidade e uso de aparelho ortodôntico. No entanto, verificou-se associação significativa entre o perfil rugoscópico das universitárias avaliadas e a realização de cirurgias bucais prévias.
\end{abstract}

Palavras-chave: Identificação humana; Odontologia Legal; Palato.

\begin{abstract}
Objective: To analyze the palatal rugae patterns of a young Brazilian adults' group, to classify this sample using the rugoscopic technique, as well as to evaluate possible differences between age group, skin color, use of orthodontic braces and oral surgeries in those surveyed. Material and Methods: Observational, classificatory, and transversal study, with a quantitative approach to palate wrinkles. The convenience sample was obtained from 30 female university students from a Dentistry course at a private educational institution. Data collection was performed using intraoral photographs of each participant's palate, highlighting palatal wrinkles with the help of Microsoft Paint ${ }^{\circledR}$, with subsequent classification of the images by a single researcher. For the classification of the university student's palatine wrinkles, the method proposed by Carrea (1937) was taken into account. The data were analyzed statistically using the Chi-Square, Fischer Exact and Likelihood Ratio tests $(\mathrm{p}=0.05)$. Results: There was a predominance of women aged 19 to 22 years $(70.0 \%)$, white $(83.3 \%)$, who used an orthodontic appliance $(83.3 \%)$ and denied having undergone any type of oral surgery (56.7\%). The study identified seven to 18 palatine wrinkles, with different shapes. Conclusion: There was a higher incidence of wrinkles directed in different directions (type IV). Palatal rugoscopy did not result in characterizations with age, ancestry and use of orthodontic braces. However, there was a significant association between the rugoscopic profile of the university students evaluated and previous oral surgeries.
\end{abstract}

Keywords: Human identification; Forensic Dentistry; Palate. 


\section{Resumen}

Objetivo: Analizar la rugosidad palatina de un grupo de jóvenes adultas brasileñas, clasificar esta muestra mediante técnica rugoscópica, así como evaluar posibles diferencias entre grupo de edad, color de piel, uso de ortodoncia y cirugías bucales en los encuestados. Material y métodos: Estudio observacional, clasificatorio y transversal, con abordaje cuantitativo de las arrugas del paladar. La muestra de conveniencia se obtuvo de 30 estudiantes universitarias del curso de Odontología en una institución privada. La recolección de datos se realizó mediante fotografías intraorales del paladar de cada participante, resaltando las arrugas palatinas con la ayuda de Microsoft Paint $\AA$, con posterior clasificación de las imágenes por un solo investigador. Para la clasificación de las arrugas palatinas de estudiantes universitarias se tuvo en cuenta el método propuesto por Carrea (1937). Los datos se analizaron estadísticamente mediante las pruebas de Chi-Cuadrado, Exacto de Fischer y Razón de Verosimilitud ( $\mathrm{p}=0,05)$. Resultados: Hubo predominio de mujeres de 19 a 22 años $(70,0 \%)$, blancas $(83,3 \%)$, que utilizaron aparato de ortodoncia $(83,3 \%)$ y negaron haberse sometido a algún tipo de cirugía oral. $(56,7 \%)$. El estudio identificó de siete a 18 arrugas palatinas, con diferentes formas. Conclusión: Hubo una mayor incidencia de arrugas dirigidas en diferentes direcciones (tipo IV). La rugoscopia palatina no resultó en caracterizaciones con edad, ascendencia y uso de aparatos de ortodoncia. Sin embargo, hubo asociación significativa entre el perfil rugoscópico de los estudiantes universitarios evaluados y cirugías bucales previas.

Palabras clave: Identificación humana; Odontología Forense; Paladar (hueso).

\section{Introdução}

A Antropologia Forense consiste na área de investigação pericial, que tem como principal objetivo estabelecer a identidade dos indivíduos, e pode ser um processo demasiadamente complexo (Poojya, Shruthi, Rajashekar \& Kaimal, 2015). A rugoscopia palatina ou palatoscopia é uma ferramenta da Antropologia, que pode ser utilizada pelo método comparativo na identificação humana (IH), tanto de indivíduos vivos como em cadáveres recentes, desde que haja registros ante mortem (AM) (Tornavoi \& Silva, 2010).

A IH, de grande relevância para a vida em sociedade, é normalmente, feita de maneira rápida e segura pela análise de documentos com foto da pessoa, como carteira nacional de habilitação $(\mathrm{CNH})$, documento de identidade, passaporte, dentre outos. Mas, em casos em que nao é possível fazer essa averiguação, pode-se realizar a identificação, por meio da identidade biológica do indivíduo, com base na análise quantitativa e qualitativa das estruturas do corpo humano (Vanrell, 2019).

Para que um método de identificação seja aplicável em processos de $\mathrm{IH}$, alguns princípios técnicos e biológicos devem ser respeitados: a unicidade (um único indivíduo deve conter determinados elementos que o diferencia de outros); a imutabilidade (os caracteres não se alteram com o tempo); a perenidade (capacidade de resistir à ação do tempo, sendo capaz de permanecer viável, inclusive após a morte); a praticabilidade (a obtenção dos registros deve ser fácil e de baixo custo); e classificabilidade (os dados obtidos devem ser arquivados, facilitando futuramente a sua localização) (Belotti et al., 2015; Vanrell, 2019).

A Odontologia Legal (OL) tem a sua indicação precípua nos casos de estado avançado de decomposição ou esqueletização cadavérica, casos em que, para a formalização do óbito no Brasil, faz-se necessária a identificação médicoodonto-legal. A identificação odontolegal é didaticamente dividida em três etapas: exame dos arcos dentários do cadáver, buscando-se levantar as particularidades; exame da documentação odontológica, coletando-se informações do tratamento efetuado; e o confronto odontolegal (Silva, 1997).

A referida identificação é realizada para a obtenção da identidade biológica individual da vítima, por meio de uma metodologia comparativa. São executadas em etapas distintas; este método necessita de registros AM, para serem comparados aos registros dos arcos dentais post mortem (PM). Os registros AM podem ser fichas clínicas, modelos de gessos, radiografias e fotografias. Já o exame antropológico, realizado para a obtenção da identidade geral do individuo, tem o intuito de se estimar a idade, etnia, sexo e estatura de um indivíduo, constitui um exemplo de metodologia reconstrutiva, sendo auxiliar no processo de identificação (Vanrell, 2019).

A rugoscopia palatina é um método da OL, em que a IH pode ser obtida pela inspeção das rugas transversais palatinas localizadas no interior da cavidade bucal (Tornavoi \& Silva, 2010). As rugas do palato são imutáveis durante a vida toda do 
indivíduo, desde antes do nascimento, tendo sua formação no terceiro mês do período embrionário e permane até certo tempo PM. Ademais, esta é uma metodologia de identificação que apresenta, mesmo em condições desfavoráveis, eficiência na sua utilização (Castro-Silva, Silva \& Veiga, 2014; Furlan, Nogueira, Demetrio \& Lolli, 2016; Lima et al., 2016; Garbin, Amaral \& Greghi, 2017).

Para alguns casos específicos, como nos de destruição dos dentes remanescentes ou vítimas desdentadas totais, a análise das rugas labiais (Silva et al., 2020) ou palatais pode ser uma importante alternativa para a IH em um contexto pericial (Bansode \& Kulkarni, 2009; Ohtani, Nishida, Chiba, Fukuda, Miyamoto \& Yoshioka, 2008). Além disso, em acidentes nos quais há a destruição das papilas dérmicas e impossibilidade do exame pelas arcadas dentárias, as rugas podem ser preservadas devido a fatores de proteção referentes aos ossos da face, estruturas moles, bem como músculos presentes ao seu redor, sendo de grande importância a sua utilização na IH (Santos, Fernandes \& Serra, 2011).

Neste contexto, os objetivos do presente estudo foram analisar a rugosidade palatina de um grupo de jovens adultas brasileiras, classificar segundo o método de Carrea (1937) esta amostra, bem como avaliar possíveis diferenças entre faixas de idade, cor da pele, uso de aparelho ortodôntico e realização de cirurgias bucais nas pesquisadas.

\section{Material e Métodos}

O presente estudo foi aprovado pelo Comitê de Ética em Pesquisa (CAAE: 57456816.4.0000.5539) de uma instituição de ensino superior (IES), localizada na região Sul do Brasil, segundo o parecer $n^{\circ} 1.627 .111 / 2016$, e todas as participantes assinaram o Termo de Consentimento para inclusão na pesquisa.

Foi realizado um estudo observacional, classificatório, transversal, com natureza quantitativa das rugas palatais, segundo o método de Carrea (1937). A amostra de conveniência da pesquisa foi composta 30 universitárias do sexo feminino, de um curso de Odontologia de IES privada da região Sul brasileira, matriculadas no quarto e quinto ano do referido curso. Todos os discentes foram convidados a participar da pesquisa, mas cerca de $60 \%$ destes, de um total de 90 formandos, recusaram-se a participar em razão da indisponibilidade do tempo e problemas gástricos.

Excluíram-se da amostra, os discentes do sexo masculino, pela pequena quantidade homens no curso. Também foram descartados, indivíduos que apresentavam lesões no palato em decorrência de traumas, inflamações, más formações ou ainda, outras anormalidades, resultantes em alterações da mucosa bucal, e obtenção inadequada da técnica de moldagem do arco superior (Garbin, Amaral \& Greghi, 2017).

A coleta de dados realizou-se no primeiro semestre de 2019, por uma única pesquisadora/observadora em ambiente clínico e seguindo todas as normas de biossegurança. Para cada acadêmica avaliada ( $n=30)$, anotou-se em uma ficha rugoscópica as variáveis idade, cor da pele autodeclarada, uso de aparelho ortodôntico e realização prévia de cirurgia bucal.

Em seguida, realizaram-se fotografias intrabucais, do palato, de cada uma das participantes, com uma câmera digital PowerShot SX60 HS (Canon Inc.®, Tóquio, Japão), e realizado um destaque das rugas do palato com uso do software Paint (Microsoft@, Redmond, Washington, EUA), e posterior análise e classificação das imagens obtidas por uma única pesquisadora/observadora que avaliou todas as imagens geradas pelas fotografias (Figuras 1 e 2). 
Figuras 1 e 2. Fotografias intrabucais com as rugosidades palatinas delimitadas $(n=30)$.

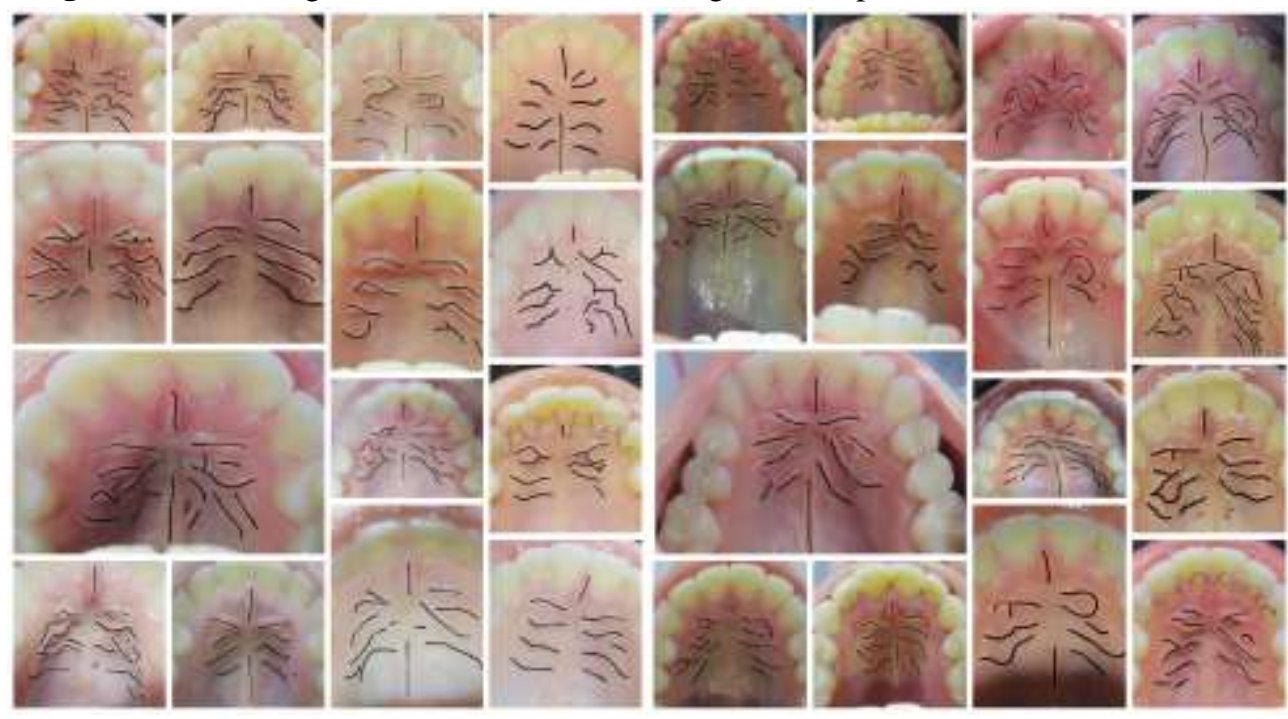

Fonte: Autores.

Para classificação das rugosidades do palato das universitárias, levaram-se em consideração os tipos principais de rugosidades descritos por Carrea (1937): rugas com direcão medial de trás para frente (tipo I); rugas com direção perpendicular à linha media (tipo II); rugas com direção medial da frente para trás (tipo III); ou rugas com direcão em sentidos variados (tipo IV).

Posteriormente, a realização das classificações de todas as fotografias das discentes pesquisadas, elaborou-se uma planilha eletrônica com informações das rugosidades do palato com uso do software Excel (Microsoft ${ }^{\circledR}$, Redmond, Washington, EUA), e informações da quantidade de rugas de cada universitária avaliada.

Os dados quantitativos obtidos no estudo foram processados no software Statistical Package for the Social Sciences (SPSS), e realizada à análise estatística descritiva e inferencial não paramétrica (Teste Qui-Quadrado, Exato de Fischer e Razão de Verossimilhança), considerando o nível de significância de 5\% (p=0,05).

\section{Resultados}

Neste estudo sobre rugosidade palatina em uma amostra de jovens adultas brasileiras, houve um predomínio de mulheres com faixa de idade de 19 a 22 anos (70,0\%), e com significante participação de acadêmicas brancas (83,3\%) (Tabela 1). Em geral, não foram encontradas imagens rugoscópicas com formas semelhantes, tipos e quantidade de rugas, tratando-se de características individuais e únicas entre as universitárias avaliadas. Segundo a utilização de aparelhos ortodônticos, um significativo uso foi verificado entre as avaliadas $(83,3 \%)$, e que negaram ter realizado qualquer cirurgia bucal até o momento $(56,7 \%)$ (Tabela 1$)$. 
Tabela 1. Análise descritiva segundo faixas de idade, cor da pele, uso de aparelho ortodôntico e realização de cirurgia bucal da amostra do estudo, $2019(\mathrm{n}=30)$.

\begin{tabular}{lcc}
\hline \multicolumn{1}{c}{ Variáveis } & n & \% \\
\hline Faixa etária & 21 & 70,0 \\
De 19 a 22 anos & 9 & 30,0 \\
De 23 a 26 anos & 3 & \\
\hline Cor da pele & 25 & 10,0 \\
Faioderma & 2 & 63,3 \\
Leucoderma & & \\
Melanoderma & 25 & 83,3 \\
\hline Uso de aparelho ortodôntico & 5 & 16,7 \\
Sim & & \\
Nega & 13 & 43,3 \\
\hline Cirurgia bucal & 17 & 56,7 \\
Sim & & \\
Nega & 3 & 23,1 \\
Qual cirurgia? & 6 & 46,2 \\
Estética & 4 & 30,8 \\
Exodontia & 13 & 100,0 \\
Exodontia e estética & $\mathbf{1 3 3}$ & $\mathbf{1 0 0 , 0}$ \\
Total & & \\
\hline Total & &
\end{tabular}

Fonte: Autores.

Encontrara-se de sete a 18 rugas no palato das universitárias, com variação nas formas: rugas em linhas, com ondulações, compostas e/ou em círculos. Segundo a classificação de Carrea (1937), houve uma leve predominância das rugas do tipo IV (33,3\%). Outras classes apresentaram menor incidência e variável de acordo com os palatos analisados (Tabela 2).

O perfil de rugosidades do palato não resultou em caracterizações com diferenças etárias, de cor da pele e uso de ortodontia prévia, no entanto verificou-se associação significativa entre o perfil rugoscópico das universitárias avaliadas e a realização de cirurgias bucais (Tabela 2).

Tabela 2. Avaliação da classe rugoscópica segundo Carrea (1937), e as variáveis de estudo nas acadêmicas de Odontologia, $2019(n=30)$.

\begin{tabular}{|c|c|c|c|c|c|c|c|c|c|}
\hline \multirow{3}{*}{ Variáveis } & \multicolumn{8}{|c|}{ Método de Carrea } & \multirow{3}{*}{ p-valor } \\
\hline & \multicolumn{2}{|c|}{$\mathbf{I}$} & \multicolumn{2}{|c|}{ II } & \multicolumn{2}{|c|}{ III } & \multicolumn{2}{|c|}{ IV } & \\
\hline & $\mathbf{n}$ & $\%$ & $\mathbf{n}$ & $\%$ & $\mathbf{n}$ & $\%$ & $\mathbf{n}$ & $\%$ & \\
\hline \multicolumn{10}{|l|}{ Faixa etária } \\
\hline De 19 a 22 anos & 2 & 66,7 & 7 & 87,5 & 5 & 55,6 & 7 & 70,0 & $0,528 * * *$ \\
\hline De 23 a 26 anos & 1 & 33,3 & 1 & 12,5 & 4 & 44,4 & 3 & 30,0 & \\
\hline \multicolumn{10}{|l|}{ Cor da pele } \\
\hline Faioderma & - & - & 1 & 12,5 & - & - & 2 & 20,0 & $* * * *$ \\
\hline Leucoderma & 2 & 66,7 & 7 & 87,5 & 8 & 88,9 & 8 & 80,0 & \\
\hline Melanoderma & 1 & 33,3 & - & - & 1 & 11,1 & - & - & \\
\hline \multicolumn{10}{|l|}{ Uso de aparelho } \\
\hline $\operatorname{Sim}$ & 3 & 100,0 & 6 & 75,0 & 9 & 100,0 & 7 & 70,0 & $0,121 * * *$ \\
\hline Nega & - & - & 2 & 25,0 & - & - & 3 & 30,0 & \\
\hline \multicolumn{10}{|l|}{ Cirurgia Bucal } \\
\hline Sim & 1 & 33,3 & 1 & 12,5 & 7 & 77,8 & 4 & 40,0 & $0,042 * * *$ \\
\hline Nega & 2 & 66,7 & 7 & 87,5 & 2 & 22,2 & 6 & 60,0 & \\
\hline Total & 3 & 100,0 & 8 & 100,0 & 9 & 100,0 & 10 & 100,0 & - \\
\hline
\end{tabular}

* Teste do Qui-quadrado/ ** Teste Exato de Fisher/ *** Razão de Verossimilhança/ **** Teste prejudicado devido ao grande número de células zeradas. Fonte: Autores. 


\section{Discussão}

As rugas do palato são estruturas anatômicas localizadas na região anterior do palato, sendo exclusivas para cada indivíduo, e podem servir como meio de IH. Alguns autores encontraram diferenças sutis nas características anatômicas das rugosidades entre diferentes grupos populacionais, ao utilizar as análises rugoscópicas de acordo com distintos sistemas de classificação (Kallianpur, Desai, Kasetty, Sudheendra \& Joshi, 2011; Shetty, Anshi Jain, Khanna, Amit Gupta \& Chowdhary, 2016). O presente estudo mostrou uma maior prevalência de rugas retas, em contraste, a uma população venezuelana, que exibiu um maior número de rugas onduladas, embora o número de sete a 18 rugas se aproxime ao encontrado por España, Paris, Florido, Arteaga e Solórzano (2010).

Entres as jovens adultas avaliadas no presente estudo, não observaram-se diferenças entre as faixas de idade e cor da pele, tanto na anatomia, quanto no quantitativo de rugas, o que corrobora com os resultados de Garbin, Amaral e Greghi (2017). Assim, entende-se que na amostra estudada, a capacidade discriminatória das rugosidades do palato foi insuficiente para se evidenciar diferenças entre faixa etária ou padrão de ancestralidade das avaliadas.

De forma semelhante, outros estudos realizados com objetivos de estabelecer correlações entre o perfil rugoscópico entre diferentes etnias e faixas de idade, não encontraram associação entre de cor da pele e idade em relação aos parâmetros biológicos das rugosidades palatinas (España et al., 2010; Kolude, Akinyele, Joshua \& Ahmed, 2016; Garbin, Amaral \& Greghi, 2017).

Ainda, comparando-se ao estudo de Garbin, Amaral \& Greghi (2017) relacionado à análise dos tipos de rugosidades do palato, observou-se maior prevalência de indivíduos tipo IV de Carrea (1937), em concordância com os resultados do presente estudo. Entretanto, houve menor predominância de indivíduos tipo III, e, neste estudo, encontrou-se menor predominância de indivíduos tipo I.

Shetty et al. (2016) investigaram o padrão de rugosidades do palato em homens e mulheres, e avaliaram suas características no pré e pós-tratamento ortodôntico. Apesar das mudanças ocorridas nas estruturas ósseas, decorrentes do tratamento, o estudo demonstra que não houve nenhuma modificação significativa nas rugosidades palatinas. Analogamente, neste estudo, não encontrou-se nenhuma variação mediante a ortodontia prévia relatada pelas avaliadas, no entanto, verificouse associação significativa com a realização de cirurgias bucais.

Ainda, no caso de pacientes que realizaram tratamento ortodôntico, a anatomia e posição das rugas do palato permanecem inalteradas, e podem ser utilizadas como um importante marcador forense. Entretanto, a utilização das medidas de comprimento das rugas, deve ser realizada com cuidado, uma vez, que pode haver alterações no tamanho das rugas de acordo com o tipo de terapia/técnica utilizada no tratamento de Ortodontia (Deepak et al., 2014; Ali, Shaikh \& Fida, 2016).

Um importante resultado do presente estudo, foi a associação encontrada entre o perfil rugoscópico e a realização de diferentes cirurgias bucais nas pesquisadas, e que também pode ser referido aos pacientes que apresentam deformidades transversais, como a constrição maxilar transversal, e recebem tratamento de expansão rápida da maxila (ERM) (Kapoor \& Miglani, 2015). Dessa forma, acredita-se que o uso das rugas do palato como identificador forense para estes indivíduos ainda gera questionamentos e necessita de novas pesquisas.

Importante ressaltar que existem poucos estudos populacionais sobre rugoscopia no Brasil, e estas pesquisas isoladas (Castro-Silva, Silva \& Veiga, 2014; Silva, Antunes, Carvalho, Soriano, Saboia \& Porto, 2019) foram realizadas na região Sudeste e Nordeste do Brasil. Portanto, este é um dos raros estudos realizados em uma amostra de adultas jovens da região Sul do país. 
No Brasil, uma prova da viabilidade deste método de identificação repousa no fato do Ministério da Aeronáutica exigir e confeccionar a identificação de cada piloto, incluindo os dados rugoscópicos como forma de facilitar a individualização destes em casos de acidentes aéreos (Paiva Modesto \& Figueira Junior, 2017).

Castellanos, Higuera, Avella, Gutiérrez \& Martínez (2007) descreveram um caso em que a identificação da vítima foi possível através da palatoscopia. Em 1993, o corpo de uma mulher foi encontrado em Cundinamarca, localizada na Colômbia. Devido ao estado em que se encontrava, não foi possível realizar a identificação pela inspeção visual. Após o início das buscas pelos desaparecidos, foram coletados os dados de uma mulher declarada desaparecida há 15 dias, em que foi disponibilizada uma prótese superior, e foi possível obter um modelo de gesso para o confronto com os dados coletados do cadáver. A técnica comparativa das rugosidades do palato foi empregada no descrito caso, ao concluir pela identificação positiva da suspeita.

Em outro exemplo, Argollo, Argollo, Argollo e Marques (2017) descreveram um caso, que mostra a eficiência da técnica rugoscópica para identificação de um cadáver carbonizado em Salvador/BA. Em meio a coleta de dados da suposta vítima, foi apresentado um prontuário odontológico onde constava a documentação ortodôntica do suspeito, incluindo os modelos de gesso, que possibilitou a análise do palato duro e suas rugosidades. Após a realização do confronto odontolegal, a técnica comparativa da rugoscopia palatina permitiu a identificação da vítima em investigação.

Um ponto discutível no método da palatoscopia é o fato de não existir um sistema universal de classificação, mas diferentes métodos classificatórios, como: Carrea (1937), Basauri (1961), Cormoy (1963) e Thomas e Kotze (1983). Sendo assim, torna-se necessário que haja a padronização de um sistema classificatório para o método rugoscópico, que possui um elevado potencial para tornar-se mais reconhecido e utilizado em âmbito forense, sendo as rugas do palato únicas, perenes, imutáveis, de fácil obtenção, e que cumprem todos os requisitos necessários para uma boa técnica de IH (Martins-dos-Santos, 1946; Patil, Patil, \& Archary, 2008).

A praticabilidade da técnica garante uma análise rugoscópica simples com um exame pericial rápido e de baixo custo, sendo esta, uma das vantagens da rugoscopia. Porém, como desvantagem da técnica, consiste o fato da dificuldade em obter dados registados em vida, sendo fundamentais para a sua utilização. Além disso, o profissional deve ser devidamente treinado, para a obtenção correta dos registros e a aplicação do método comparativo (Brígido, 2018).

As rugas do palato não podem ser alteradas com o tempo, sendo assim é uma técnica confiável e segura que preenche os requisitos técnicos e biológicos para ser utilizada na IH. Porém, outro fator sensível na utilização da palatoscopia é a interpretação do observador que pode ocasionar diferenças na análise comparativa, bem como a utilização de métodos indiretos (fotografias), como a realizada nesta pesquisa. Logo, novos estudos são necessários para elucidar a magnitude das rugas palatinas na IH, bem como as possíveis diferenças entre o padrão rugoscópico entre indivíduos de diferentes faixas etárias, cor de peles e características bucais, ortodônticas e cirúrgicas.

\section{Conclusão}

No presente estudo, houve uma maior incidência de rugas em sentidos variados (tipo IV) e pequena ocorrência de rugas com direção medial de trás para frente (tipo I), segundo a classificação proposta por Carrea. A rugoscopia palatina não resultou em caracterizações com diferenças etárias, de cor da pele (ancestralidade) e uso de aparelho ortodôntico, no entanto verificou-se associação significativa entre o perfil rugoscópico das jovens adultas brasileiras e a realização de cirurgias bucais prévias. Pode-se ainda, concluir que o método rugoscópico, mesmo sendo pouco empregado e conhecido, pode ser considerado viável como técnica auxiliar ou principal no processo de identificação forense.

\section{Conflito de Interesse}

Todos os autores declaram não haver conflito de interesse. 


\section{Referências}

Ali, B., Shaikh, A., \& Fida, M. (2016). Stability of palatal rugae as a forensic marker in orthodontically treated cases. Journal of Forensic Sciences, 61(5), $1351-1355$

Argollo, S. D. P., Argollo, B. P., Argollo, P. A. N., \& Marques, J. A. M. (2017). Utilização da rugoscopia palatina para identificação de corpo carbonizado: Relato de caso pericial. Revista Brasileira de Odontologia Legal, 4(1), 107-113.

Basauri, C. (1961). Forensic odontology and identification. Int Crim Police Rev, 16, 45-51.

Bansode, S. C., \& Kulkarni, M. M. (2009). Importance of palatal rugae in individual identification. Journal of Forensic Dental Sciences, 1(2), 77-81.

Belotti, L., Rabbi, R., Pereira, S. D. R., Barbosa, R. S., Carvalho, K. S., \& Pacheco, K. T. S. (2015). É possível identificar positivamente um corpo carbonizado somente por dois dentes? Relato de um caso pericial. Revista Brasileira de Odontologia Legal, 2(2), $105-115$.

Brígido, J. A. (2018). Rugoscopia palatina na Odontologia Forense. Revista Diálogos Acadêmicos, 7(2), 15-19.

Carrea, J. U. (1937). La identificación humana por las rugosidades palatinas. Revista Orthodontica, 1, 3-23.

Castellanos, D. C. A, Higuera, L. F. H, Avella, A. M. H., Gutiérrez, A. P. P., \& Martínez, J. A. C. (2007). Identificación positiva por medio del uso de la rugoscopia en un município de Cundinamarca (Colombia): Reporte de caso. Acta Odontológica Venezolana, 45(3), $446-449$.

Castro-Silva, I. L., Silva, O. M. L., \& Veiga, B. M. C. (2014). Uso da rugoscopia palatina como ferramenta biométrica: Um estudo populacional em NiteróiRJ, Brasil. Revista de Odontologia da UNESP, 43(3), 203-208.

Cormoy, J. P. (1973). La rugoscopie. Rev Chirurgien Dentiste France, 59-60.

Deepak, V., Malgaonkar, N. I., Shah, N. K., Nasser, A. S, Dagrus, K., \& Bassle, T. (2014). Palatal rugae patterns in orthodontically treated cases, are they a reliable forensic marker? Journal of International Oral Health, 6(5), 89-95.

España, L., Paris, A., Florido, R., Arteaga, F., \& Solórzano, E. (2010). Estudio de las características individualizantes de las rugas palatinas. Caso: bomberos de la Universidad de los Andes Mérida-Venezuela. Cuadernos de Medicina Forense, 16(4), 199-204.

Furlan, A. C. K., Nogueira, B. S., Demetrio, A. T. W., \& Lolli, L. F. (2016). Validação do método de carrea na região noroeste do estado do Paraná, Brasil. Revista Brasileira de Odontologia Legal, 3(1), 15-23.

Garbin, C. A. S., Amaral, M. A., \& Greghi, R. S. S. (2017). Análise e classificação da rugosidade palatina em uma população brasileira. Revista Brasileira de Odontologia Legal, 4(3), 48-56.

Kallianpur, S., Desai, A., Kasetty, S., Sudheendra, U., \& Joshi, P. (2011). An anthropometric analysis of facial height, arch length, and palatal rugae in the Indian and Nepalese population. Journal of Forensic Dental Sciences, 3, 33-37.

Kapoor, P., \& Miglani, R. (2015). Transverse changes in lateral and medial aspects of palatal rugae after mid palatal expansion: A pilot study. Journal of Forensic Dental Sciences, 7(1), 8-13.

Kolude, B., Akinyele, A., Joshua, O. T., \& Ahmed, L. (2016). Ethnic and gender comparison of rugae patterns among clinical dental trainees in Ibadan, Nigeria. The Pan African Medical Journal, 20(23), 204.

Lima, M. V. F. N., Costa, G. M., Silva, V. B., Nascimento, M. R., Moraes, H. H., \& Lucena, E. E. S. (2016). Verificação da praticabilidade e da unicidade na queiloscopia e na palatoscopia como métodos de identificação humana. Revista Brasileira de Odontologia Legal, 3(1), 5-14.

Martins-dos-Santos, G. (1946). Ficha odonto-rugopalatina. Revista Brasileira de Odontologia, 4(1), 36-42.

Ohtani, M., Nishida, N., Chiba, T., Fukuda, M., Miyamoto, Y., \& Yoshioka., N. (2008). Indication and limitations of using palatal rugae for personal identification in edentulous cases. Forensic Science International. 176(2-3):178-182.

Paiva Modesto, T. O., \& Figueira Junior, E. (2014). Identificação humana através da rugoscopia palatina. Revista Interdisciplinar de Direito, 11(2), 461-472.

Patil, M. S., Patil, S. B., \& Achary, A. B. (2008). Palatine rugae and their significance in clinical dentistry: A review of the literature. Journal of the American Dental Association, 139(11), 1471-1478.

Poojya, R., Shruthi, C. S., Rajashekar, V. M., \& Kaimal, A. (2015). Palatal rugae patterns in edentulous cases, are they a reliable forensic marker? International Journal of Biomedical Science. 11(3):109-112.

Santos, K. C., Fernandes, C. M., \& Serra, M. C. (2011). Evaluation of a digital methodology for human identification using palatal rugoscopy. Brazilian Journal of Oral Sciences, 10 (3), 199-203.

Shetty, D., Anshi Jain, A. J., Khanna, K. S., Amit Gupta, N. P., \& Chowdhary, M. (2016). Assessment of palatal rugae pattern and their reproducibility for application in forensic analysis. Journal of Forensic Dental Sciences, 5(2), 106-109.

Silva, M. (1997). Compêndio de Odontologia Legal. Medsi.

Silva, F. L. M., Antunes, A. A., Carvalho, M. V. D., Soriano, E. P., Saboia, R. S. C., \& Porto, G. G. (2019). Avaliação do padrão morfológico de rugoscopia palatal em uma população brasileira. Revista Brasileira de Odontologia Legal, 6(2), 2-12. 
Research, Society and Development, v. 10, n. 1, e46810111743, 2021

(CC BY 4.0) | ISSN 2525-3409 | DOI: http://dx.doi.org/10.33448/rsd-v10i1.11743

Silva, A. V., Costa, A. L. P., Silva, M. L. C. A., Oliveira, M. B., Paranhos, L. R., Franco, A. Marques, J. A. M., \& Musse, J. (2020). Estudo anatômico do padrão labial entre mães e filhas do Nordeste brasileiro. Research, Society and Development, 9(11), e94691110545.

Thomas, C. J., \& Kotze, T. J. (1983). The palatal ruga pattern: A new classification. Journal of the Dental Association of South Africa, $38,153-157$.

Tornavoi, D. C., \& Silva, R. H. A. (2010). Rugoscopia palatina e a aplicabilidade na identificação humana em Odontologia Legal: Revisão de literatura. Saúde, Ética \& Justiça, 15(1), 28-34.

Vanrell, J. P. (2019). Odontologia Legal e Antropologia Forense. (3a ed.), Guanabara Koogan. 